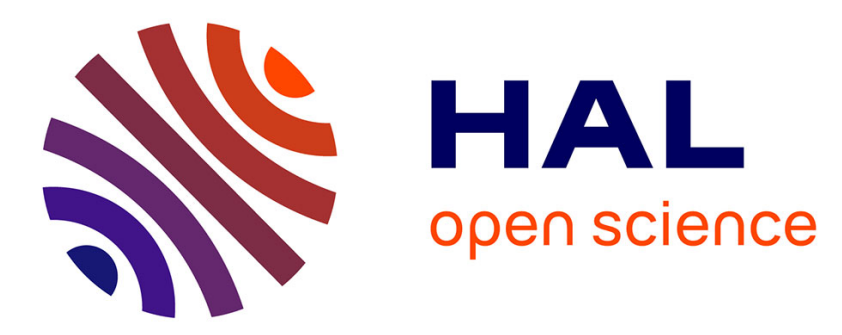

\title{
High temperature quantum tunnelling of magnetization and thousand kelvin anisotropy barrier in a Dy(2) single-molecule magnet
}

Haipeng Wu, Min Li, Zhengqiang Xia, Vincent Montigaud, Olivier Cador, Boris Le Guennic, Hongshan Ke, Wenyuan Wang, Gang Xie, Sanping Chen, et al.

\section{To cite this version:}

Haipeng Wu, Min Li, Zhengqiang Xia, Vincent Montigaud, Olivier Cador, et al.. High temperature quantum tunnelling of magnetization and thousand kelvin anisotropy barrier in a Dy (2) single-molecule magnet. Chemical Communications, 2021, 57 (57), pp.371-374. 10.1039/d0cc06993k . hal-03102116

\author{
HAL Id: hal-03102116 \\ https://hal.science/hal-03102116
}

Submitted on 23 Feb 2021

HAL is a multi-disciplinary open access archive for the deposit and dissemination of scientific research documents, whether they are published or not. The documents may come from teaching and research institutions in France or abroad, or from public or private research centers.
L'archive ouverte pluridisciplinaire HAL, est destinée au dépôt et à la diffusion de documents scientifiques de niveau recherche, publiés ou non, émanant des établissements d'enseignement et de recherche français ou étrangers, des laboratoires publics ou privés. 


\section{High temperature quantum tunnelling of magnetization and thousand kelvin anisotropy barrier in a $\mathrm{Dy}_{2}$ single-molecule magnet}

Received 00th January 20xx, Accepted 00th January 20xx

DOI: $10.1039 / x 0 x \times 00000 x$

\author{
Haipeng Wu, ${ }^{\ddagger a, c}$ Min Li, ${ }^{\ddagger a, c}$ Zhengqiang Xia, ${ }^{* a}$ Vincent Montigaud, ${ }^{\mathrm{b}}$ Olivier Cador, ${ }^{* b}$ \\ Boris Le Guennic, ${ }^{* b}$ Hongshan Ke, ${ }^{a}$ Wenyuan Wang, ${ }^{a}$ Gang Xie, ${ }^{a}$ Sanping Chen*a and Shengli Gao ${ }^{a}$
}

\begin{abstract}
We report here a dinuclear Dy"II iodine-bridged single-molecule magnet self-assembled by cis/trans coordination chemistry that displays a large anisotropy barrier of $c a .1300 \mathrm{~K}$ and a hysteresis opening temperature of $16 \mathrm{~K}$. High temperature quantum tunnelling of magnetization is observed up to $56 \mathrm{~K}$ in zero-field and explained by the combination of large anisotropy barrier and the local transverse field at the trans site. The results provide a model for thorough understanding of the effect of electronic structure on the magnetic behavior of lanthanide complexes.
\end{abstract}

The strong magnetic anisotropy of lanthanide complexes often induces single-molecule magnets (SMM) behavior, ${ }^{1}$ which spurs their various applications in prototype magnetic devices. ${ }^{2,3}$ Among the lanthanide based SMMs, Dy'll' is acclaimed as an unrivalled candidate owing to the strongly oblate spheroidal shape of its electron density and the presence of Kramers doublets. ${ }^{4}$ The anisotropy of Dy $\mathrm{y}^{\text {III }}$ is therefore enhanced in axial coordination environments. ${ }^{5,6}$ The design of Dy'll complexes with low coordination numbers presents a particularly efficient avenue to realize high degree of axiality but remains quite synthetically challenging. The pitfall in lanthanide chemistry is the significant quantum tunneling of the magnetization (QTM)

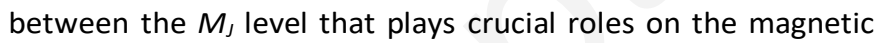
properties. ${ }^{7}$ The existence of fast QTM often causes the reduction or even loss of memory effect of the magnetization at zero field, limiting their potential applications. ${ }^{8,9}$ But the QTM can be reduced by consideration of the local symmetry around lanthanide ions. ${ }^{10}$ It has been shown that high CF symmetries, such as $C_{\infty v}, D_{4 \mathrm{~d}}, S_{8}, D_{5 \mathrm{~h}}, D_{6 \mathrm{~d}}$, and $D_{\infty \mathrm{h}}$, etc. eliminate transverse CFs that enlarge CF and reduce QTM. ${ }^{10}$ However, in a real

a. Key Laboratory of Synthetic and Natural Functional Molecule Chemistry of Ministry of Education, College of Chemistry and Materials Science, Northwest University, Xi'an, 710127, China

E-mail: northwindy@126.com; sanpingchen@126.com.

b. Univ Rennes, CNRS, ISCR (Institut des Sciences Chimiques de Rennes) -UMR 6226, F-35000 Rennes, France

E-mail: olivier.cador@univ-rennes1.fr; boris.leguennic@univ-rennes1.fr.

Department of Chemistry and Chemical Engineering, Jinzhong University, Jinzhong, 030619, China

+These authors contributed equally to this work.

Electronic Supplementary Information (ESI) available: Synthetic details, structural data, additional magnetic figures and $a b$ initio studies. CCDC 1901845. For ESI and crystallographic data in CIF or other electronic format see DOI: 10.1039/x0xx00000x system, the symmetry is most likely to be lower than ideal, and the high CF symmetry is most obtained from more coordinatively saturated molecules instead of low-coordinate systems. ${ }^{11}$ How to control structural factors and take advantage of the symmetry strategies to suppress QTM while maintaining the magnetic axiality in low-coordinate systems is still a current challenge that needs to be further explored.

Octahedral six-coordinate complexes, as the threshold of the regular coordination number (7-12) of lanthanide complexes towards the low-coordinate ones and with a well-defined axial and equatorial distribution, are excellent candidates to realize performant SMMs. Increasing efforts have been made to understand magnetic relaxation and optimize electrostatic distribution of Dy"I in pseudo-octahedral geometry. ${ }^{12-17}$ We have recently reported the cis/trans stereoisomers in a distorted octahedral bromine-bridged Dy"l dimer $\left[\mathrm{Dy}\left(\mathrm{Cy}_{3} \mathrm{PO}\right)_{2}(\mu-\mathrm{Br})(\mathrm{Br})_{2}\right]_{2} \cdot 2 \mathrm{C}_{7} \mathrm{H}_{8}\left(\mathbf{1} \cdot 2 \mathrm{C}_{7} \mathrm{H}_{8}\right)$ with a large effective barrier of $684 \mathrm{~K} .{ }^{15}$ Interestingly, it is characterized by the very weak magnetic interaction between Dy ${ }^{\text {III }}$ ions as a consequence of the mutual orthogonal anisotropy arrangement and the bridging bromide ions. In this system the magnetic relaxation is dominated by the strong single ion anisotropy of the trans$\mathrm{Br}_{4} \mathrm{O}_{2}$ site. ${ }^{15}$ We have decided to take advantage of this situation as we go down the periodic table: to substitute bromine with iodine. Weaker coordinating anions such as iodine 1) minimize the in-plane CF at trans site and, in principle, should maximize the axial anisotropy 2) minimize the superexchange interaction between Dy'll centers. It is striking that the energy barrier (ca. $1300 \mathrm{~K})$ of the new dinuclear Dyll' SMM $\left[\mathrm{Dy}\left(\mathrm{Cy}_{3} \mathrm{PO}\right)_{2}(\mu-\right.$ I) $\left(\mathrm{I}_{2}\right]_{2} \cdot 4 \mathrm{C}_{7} \mathrm{H}_{8} \quad\left(2 \cdot 4 \mathrm{C}_{7} \mathrm{H}_{8}\right)$ is the highest among the reported polymetallic complexes obtained via conventional coordination chemistry approaches while QTM is visible up to $c a .56 \mathrm{~K}$. Solidstate magnetic site dilution and ab-initio calculations allow rationalizing these prominent features.

The addition of $\mathrm{Dyl}_{3}$ and $\mathrm{Cy}_{3} \mathrm{PO}$ at a 1:2 molar ratio in ethyl ether produced a white suspension solution. The volatiles were removed under reduced pressure and faint yellow crystals of $2 \cdot 4 \mathrm{C}_{7} \mathrm{H}_{8}$ were obtained by recrystallization from toluene (Scheme S1). It crystallizes in the monoclinic space group of $P 2_{1} /$ n (Table S1). The asymmetric unit contains two unique stereoisomeric $\left[\mathrm{Dy}\left(\mathrm{Cy}_{3} \mathrm{PO}\right)_{2} \mathrm{I}_{4}\right]$ molecules with metal ions 


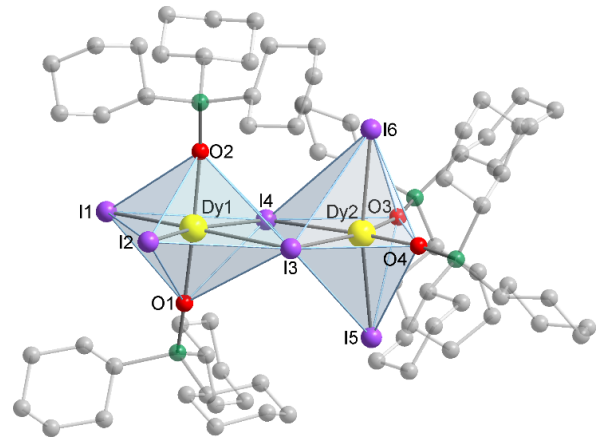

Fig. 1 Molecular structure of 2. Hydrogen atoms are omitted for clarity. For unlabeled atoms: $\mathrm{P}$ green, and $\mathrm{C}$ grey

bridged by two $\mu_{2}-\mathrm{I}$ ions and each Dy'll center is hexacoordinated adopting a distorted octahedral geometry (Fig. 1). Dy1 is ligated by two strong $\mathrm{Cy}_{3} \mathrm{PO}$ ligands in trans position which are in cis position for Dy2. The Dy-O distances (2.172-2.195 ̊) are considerably shorter than Dy-I bonds (2.985-3.192 A) (Table S2). Consequently, the coordination polyhedra are strongly distorted with CShMs values for $O_{\mathrm{h}}$ symmetry at 2.79 and 2.56 (Table S3). ${ }^{18}$ In $\mathbf{1}$, the distances are equivalent for Dy-O (2.188-2.199 Å) but much shorter for Dy$\operatorname{Br}(2.735-2.950 \AA)$ and less distorted polyhedra were observed (CShMs values at 1.921 and 1.342). ${ }^{15}$ The intramolecular Dy-Dy distance ( $4.80 \AA$ ) is longer than in 1 (4.46 ̊) while maintaining the same Dy-X-Dy angles. Within the lattice, the shortest intermolecular Dy...Dy distance is $10.661 \AA$ (Fig. S1).

Static magnetic susceptibility measurements on $2 \cdot 4 \mathrm{C}_{7} \mathrm{H}_{8}$ are carried at 1000 Oe between 2 to $300 \mathrm{~K}$. The room-temperature $\chi_{\mathrm{M}} T$ value is equal to $27.71 \mathrm{~cm}^{3} \mathrm{~K} \mathrm{~mol}^{-1}$ in agreement with the expected values for two free Dy"lI ions $\left(28.34 \mathrm{~cm}^{3} \mathrm{~K} \mathrm{~mol}^{-1}\right.$ ) (Fig. S4). $\chi_{\mathrm{M}} T$ decreases monotonically on cooling down with a low temperature limit that is close to $24.01 \mathrm{~cm}^{3} \mathrm{~K} \mathrm{~mol}^{-1}$. A small drop below $5 \mathrm{~K}$ is visible with a value of $22.95 \mathrm{~cm}^{3} \mathrm{~K} \mathrm{~mol}^{-1}$ at $2 \mathrm{~K}$. At this stage, there is no evidence of interactions (dipolar and/or superexchange) between Dy"ll magnetic moments. The $M$ vs. $H$ curve at $2 \mathrm{~K}$ saturates at value close to $10 \mathrm{~N} \beta$ in agreement with the stabilization of the $M_{J}= \pm 15 / 2$ Ising component of the ${ }^{6} H_{15 / 2}$ ground state multiplet (Fig. S4). The slow relaxation of the magnetization is evidenced through ac susceptibility measurements. A maximum shows up on the out-of-phase component of the ac susceptibility below $70 \mathrm{~K}$ in zero external dc field (Fig. 2a and Fig. S5). The maximum shifts to low frequency on lowering the temperature but becomes temperature independent below $56 \mathrm{~K}$, displaying a tunneling frequency of $412 \mathrm{~Hz}$. At each temperature, the frequency dependence of the ac signals is analyzed quantitatively with an extended Debye model (Fig. S6, Table S4). ${ }^{19}$ Important information are extracted at this stage: 1) A narrow distribution of the relaxation times $(\alpha)$ of $0.05-0.18$ is obtained, in agreement with a single relaxation process 2 ) The relaxation time follows a Arrhenius law combined with a thermally independent process $\left(\tau^{1}=\tau_{\mathrm{QTM}}{ }^{-1}+\tau_{0}{ }^{-1} \exp \left(-U_{\text {eff }} / T\right)\right)$ with $U_{\text {eff }}=1290$ $\mathrm{K}, \tau_{0}=1.26 \times 10^{-12} \mathrm{~s}$ and $\tau_{\mathrm{QTM}}=3.88 \times 10^{-4} \mathrm{~s}$ (Fig. 2b) 3) The nonrelaxing fraction $\left(\chi_{\mathrm{S}} / \chi_{\mathrm{T}}\right)$ is close to $40 \%$ in all the temperature range (Table S4). This last characteristic suggests that only one
Dy involved. Clearly, the energy barrier jumped from $\sim 700 \mathrm{~K}$ in $\mathbf{1} \cdot 2 \mathrm{C}_{7} \mathrm{H}_{8}$ up to $\sim 1300 \mathrm{~K}$ in $\mathbf{2} \cdot 4 \mathrm{C}_{7} \mathrm{H}_{8} .{ }^{15}$ It proves that our approach which consists in minimizing $\mathrm{CF}$ in the plane rather than maximizing it along one axis is valid for Dy"l'. However, QTM in 2. $4 \mathrm{C}_{7} \mathrm{H}_{8}$ is faster than in $\mathbf{1} \cdot 2 \mathrm{C}_{7} \mathrm{H}_{8}$ (Fig. S7). ${ }^{15}$ The application of an external dc field annihilates the thermally independent process and shifts the relaxation process to lower frequencies (Fig. S8). The relaxation becomes so slow on cooling that Zero-Field Cooled (ZFC) and Field-Cooled (FC) curves measured at a sweep rate of $0.5 \mathrm{~K} \mathrm{~min}^{-1}$ diverge at $12 \mathrm{~K}$ that reveal the onset of the memory effect (Fig. 2c). On Fig. $\mathrm{S} 8$ the relaxation is the slowest at $1 \mathrm{kOe}$ and the frequency dependence at various temperatures of the ac signals has been recorded at this optimum field (Fig. S9) and analyzed in the framework of the generalized Debye model (Table S5). The non-relaxing fraction remains close to $40 \%$ and now the Raman process $\left(\tau^{1}=\mathrm{CT}^{n}\right)$ becomes the fastest at low temperature. The best fit is obtained with $\mathrm{C}=1.04 \times 10^{-4} \mathrm{~s}^{-1} \mathrm{~K}^{-n}, n=3.31, U_{\text {eff }}=1323 \mathrm{~K}, \tau_{0}=7.3 \times 10^{-12} \mathrm{~s}$ (Fig. 2b). As expected, $\tau_{0}$ and $U_{\text {eff }}$ are almost unchanged between zero external dc field and 1 kOe while QTM is too slow to be numerically extracted.

The disappearance of QTM at low temperature with the magnetic field is common in Dy"l' SMMs. ${ }^{20}$ Hyperfine coupling, dipolar and/or superexchange interactions are known to be the sources of this thermally independent effect. ${ }^{21-23}$ Dilution in a diamagnetic matrix and isotopic enrichment are standard tools employed to eliminate QTM. ${ }^{24,25}$ However, in polynuclear complexes, a fortiori in dimers, the doping with non-magnetic metal ion ( $\left.\mathrm{Y}^{\prime \prime \prime}\right)$ may result in either $\mathrm{Dy}_{2}$ complexes within a matrix of diamagnetic dimers or heteronuclear Dy- $Y$ units. In the present case, $5 \%$ of Dy $\mathrm{y}^{\prime \prime \prime}$ ion has been injected in the diamagnetic YIII matrix to yield 2@Y with a final ratio Dy/Y: 1/15.2. The magnetic investigation in zero external field on 2@Y reveals a low frequency shift of $\chi_{M}$ " peak at low temperature compared with $2 \cdot 4 \mathrm{C}_{7} \mathrm{H}_{8}$ (Fig. S10). The QTM is then efficiently released by the dilution. Remarkably, the characteristic relaxation times superimpose with those of the condensed $2 \cdot 4 \mathrm{C}_{7} \mathrm{H}_{8}$ at 1 kOe (Table S6, Fig. 2b). Here again, $40 \%$ of the magnetic moment is non relaxing. The best fit is obtained with Raman+Orbach processes $\left(U_{\text {eff }}=1311 \mathrm{~K}, \tau_{0}=1.08 \times 10^{-12} \mathrm{~s}\right.$, $\left.\mathrm{C}=6.69 \times 10^{-4} \mathrm{~s}^{-1} \mathrm{~K}^{-n}, n=2.82\right)$. The dilution experiment indicates that intramolecular and/or intermolecular magnetic interactions are highly responsible for the prevalence of fast tunneling in $\mathbf{2} \cdot 4 \mathrm{C}_{7} \mathrm{H}_{8}$. However, there is no possible discrimination between the two types of dilution since we cannot dilute magnetic dimers into a diamagnetic matrix in frozen solution with ensuring the integrity of the complex in solution.

In-field magnetic hysteresis is clearly observed for $\mathbf{2} \cdot 4 \mathrm{C}_{7} \mathrm{H}_{8}$ up to $14 \mathrm{~K}$, for a sweep rate of $0.12 \mathrm{kOe} \mathrm{s}^{-1}$ (Fig. S11) which matches with the divergence of ZFC-FC curves at $12 \mathrm{~K}$. The closing at the origin whatever the temperature is clearly due to the QTM fast relaxation. On contrary, the magnetic hysteresis curves for 2@Y is open at the origin up to $16 \mathrm{~K}$ (Fig. S12). The normalized hysteresis loops are represented for both $2 @ Y$ and $2 \cdot 4 \mathrm{C}_{7} \mathrm{H}_{8}$ at 2 $\mathrm{K}$ on Fig. $2 \mathrm{~d}$ highlighting remanence $(24 \%$ of saturation 

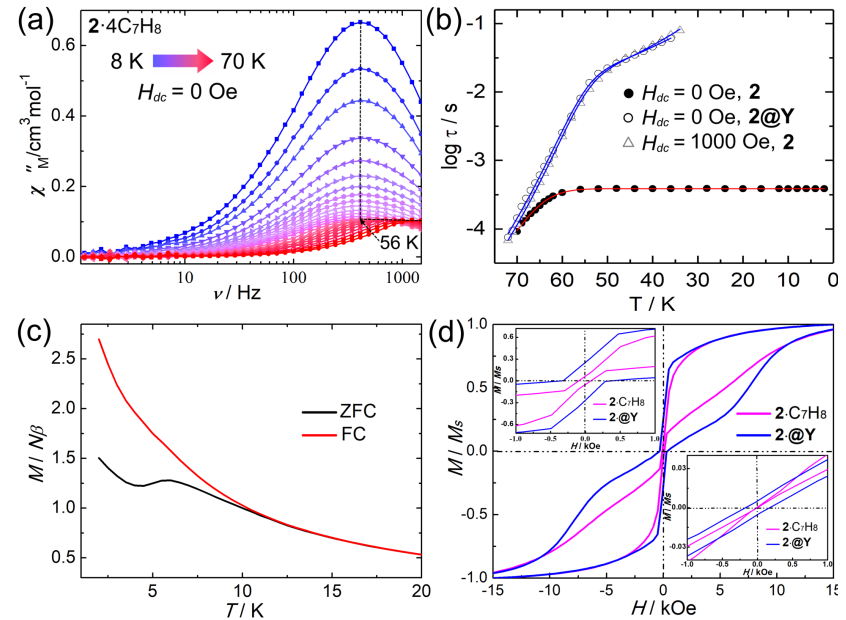

Fig. 2 (a) Frequency-dependent out-of-phase $\chi^{\prime \prime} M$ ac susceptibility signals for $\mathbf{2}$ at zero external dc field. (b) Temperature dependences of the relaxation time for $\mathbf{2}$ at zero external dc field $(\bullet) \mathbf{2}$ at $1 \mathrm{kOe}(\triangle)$ and $\mathbf{2} @ Y$ at zero external dc field $(O)$ with the best fitted curves: in red QTM+Orbach and in blue Raman+Orbach. (c) ZFC-FC magnetization curves at $2 \mathrm{kOe}$ for $2 \cdot 4 \mathrm{C}_{7} \mathrm{H}_{8}$ at a sweep rate of $0.5 \mathrm{~K} \mathrm{~min}^{-1}$. (d) Normalized $\mathrm{M}$ vs. $\mathrm{H}$ hysteresis profiles of $\mathbf{2} @ \mathbf{Y}$ and $\mathbf{2}$ at $2 \mathrm{~K}$. In inset: top left) zoom at the origin of the $2 \mathrm{~K}$ hysteresis; bottom right) zoom at the origin of the $16 \mathrm{~K}$ hysteresis.

magnetization) and coercivity (360 Oe) achieved through the dilution.

Ab-initio Complete Active-Space Self-Consistent Field (CASSCF) calculations were performed on the X-ray structure of 2, using the MOLCAS 8.2 and SINGLE_ANISO programs (see Supporting Information for computational details). Calculations were carried out on each asymmetric Dy'll center of the dimer unit (namely Dy1/Dy2) while the second metal ion was replaced by a diamagnetic $Y^{\prime \prime I}$ ion. The resulting energy spectrum and $g$ tensors ( $\left.g_{\mathrm{Xx}}, g_{\mathrm{Yy}}, g_{\mathrm{zz}}\right)$ of the eight lowest Kramers Doublets (KD) of $\mathbf{2}$ are reported in Table S7. Dy1 and Dy2 show a strong ground state anisotropy with a main $g_{z z}$ component at 19.9 for Dy 1 and only 19.1 for Dy2 $\left(g_{\mathrm{Xx}}=g_{\mathrm{YY}}=0.00\right.$ for Dy1; $g_{\mathrm{Xx}}=0.03$ and $g_{\mathrm{YY}}=0.07$ for Dy2). The orientations of the magnetic axes for the KD ground states are reported in Fig. 3. Due to the large intermolecular Dy-Dy distances (10.66 ̊̊), only intramolecular dipolar magnetic interactions are calculated. The intramolecular dipolar term was computed in the effective spin $\tilde{S}=1 / 2$ formalism using the POLY_ANISO routine (see experimental section for details). ${ }^{26,27}$ The resulting intramolecular dipolar constant is $J=-0.012 \mathrm{~cm}^{-1}$. This very weak antiferromagnetic coupling is explained by the orthogonal configuration of the anisotropy axes in the KD ground states $\left(92^{\circ}\right)$. In this frame, the computed molar magnetic susceptibility and magnetization curve at $2 \mathrm{~K}$ are in good agreement with experimental data (Fig. S4).

The electrostatic potential (Fig. 3), computed from the $a b$ initio LoProp charges using the home-made software CAMMEL, ${ }^{28,29}$ governs the Dy'll anisotropy axis orientation that follows the most negative potential induced by the $\mathrm{O}$ atoms of the $\mathrm{Cy}_{3} \mathrm{PO}$ ligands (Fig. 3 and Fig. S13). The energy spectra of the ground state multiplet ${ }^{6} H_{15 / 2}$ for each Dy'll ions are reported in Fig. 3. First, the total splitting is twice larger for Dy1 (1365 K) than for Dy2 ( $685 \mathrm{~K})$. Second, the gap between the ground state KD and the first excited KD state is four times larger for Dy1 (488
K) than for Dy2 $(122 \mathrm{~K})$. The computed transition moments between each state (Fig. 3) for both Dy1 and Dy2 show two drastically different relaxation pathways. For Dy2, the non-zero transversal components of the ground state $g$-tensor and a mixed low-lying $1^{\text {st }}$ excited state (Table S8) lead to sizable QTM and thermally assisted QTM suggesting a fast relaxation mechanism. On the contrary for Dy1, the strong Ising-type anisotropy of the ground and $1^{\text {st }}$ excited state (Table S8) results in a relaxation path through the third excited state at $1165 \mathrm{~K}$ (Fig. 3), which corresponds to the effective energy barrier (Orbach) in almost perfect agreement with the experiment $(\sim 1300 \mathrm{~K})$. The computed results support the rationale that at high temperature $\mathbf{2}$ relaxes via an over-barrier (Orbach) mechanism associated with the large single ion anisotropy of the trans-Dyl ${ }_{4} \mathrm{O}_{2}$ site.

It is noticed that the change of the ancillary halogen donors connecting Dy'll is shown not only to induce differences in the octahedrally distorted $\mathrm{DyX}_{4} \mathrm{O}_{2}$ coordination sphere that adjusts the magnetic anisotropy and the superexchange interaction but also to modify the packing and intermolecular dipolar interactions. The principal axes of monoclinic $\mathbf{2}$ are canted by $45^{\circ}$ between neighboring molecules. Whereas 1 crystallizes in the triclinic $P^{\mathrm{I}}$ space group ${ }^{15}$ without a canted angle between two independent molecules (Fig. S14). Despite there is no possible to discrimination the origin of QTM (intramolecular vs. intermolecular processes), the large tilting of the principle axes between neighboring molecules should enhance the transverse components of fluctuating magnetic fields, thus improving tunneling of the magnetization in $\mathbf{2}$.

In fact, $2 \cdot 4 \mathrm{C}_{7} \mathrm{H}_{8}$ displays the best performance of sixcoordinate SMMs with an effective barrier of up to $1300 \mathrm{~K}$ and hysteresis loops open up to $16 \mathrm{~K}$ (Table S9). This strong anisotropy combined with weak coercivity appears to be a common feature in the limited reported examples of $D_{3 \mathrm{~h}}$ and $O_{\mathrm{h}}$ geometries. ${ }^{12-17,30-32}$ Compared with two widely studied families of high-performance pentagonal bipyramidal and the metallocene dysprosium single-ion magnets, ${ }^{33-38}$ it has been found that higher charge symmetry and weaker thermal vibration of atoms favor stronger coercivity. For the reported trigonal bipyramidal and octahedral complexes, 12-17,30-32 the uneven and the monodentate coordinated transverse sites

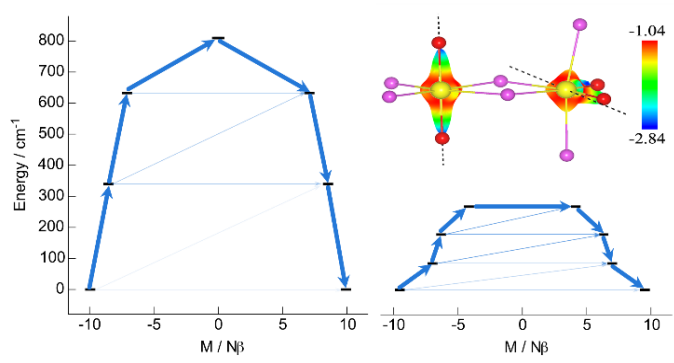

Fig. 3 Computed magnetization blocking barriers of Dy1 (left) and Dy2 (right) of 2. The four lowest KDs (black lines) are represented according to their magnetic moment along the main magnetic axis. The arrows are drawn according to the mean values of the transversal matrix element of the transition magnetic moment. The total electrostatic potential at $1.6 \AA$ of each Dy center is also represented (top right). 
diminish the CF symmetry and increase the molecular flexibility, which contributes to the off-diagonal elements of the matrix representations and thereby enhancing QTM.

In summary, we observed a large anisotropy barrier of 1300 $\mathrm{K}$ and magnetic hysteresis opened up to $16 \mathrm{~K}$ in a dimetallic octahedral Dy'II system along with significant QTM up to $56 \mathrm{~K}$. Strong QTM in the presence of large barrier is associated with the magnetic environment around Dy1. The cis/trans arrangement of strong $\mathrm{C}_{3} \mathrm{PO}$ moieties combined with weak iodine ligands around the Dy"ll centers produces two drastically different crystal fields. The exceptional properties originate from the combination of large single-ion anisotropy at trans site and cancelation of magnetic interactions through nearorthogonality of the magnetic axes. The magnetic dilution experiment proved that the dipolar coupling accounts for the significant zero-field QTM. Comparative analysis of dysprosium complexes with different configurations suggests that the synthesis of high axial low coordination systems with uniform molecule anisotropy orientations, high charge symmetry and restricted molecular vibrations are expected to achieve a qualitatively new breakthrough for the development of SMMs.

We gratefully acknowledge financial support from the National Natural Science Foundation of China (Grant no. 21673180, 21727805, 21803042 and 21973074), the Natural Science Basic Research Program of Shaanxi (Grant No. 2017JZ002, 2018JM5180 and 2019JQ-067) and the 64th China Postdoctoral Science Foundation Funded Project (2018M643706). V. M. is thankful to ERC (project no. 725184) for funding. B. L. G. and V. M. thank the French GENCI/IDRISCINES center for high-performance computing resources.

\section{Conflicts of interest}

There are no conflicts to declare.

\section{Notes and references}

1 D. N. Woodruff, R. E. Winpenny and R. A. Layfield, Chem. Rev., 2013, 113, 5110-5148.

2 R. Vincent, S. Klyatskaya, M. Ruben, W. Wernsdorfer and F. Balestro, Nature, 2012, 488, 357-360.

3 S. Thiele, F. Balestro, R. Ballou, S. Klyatskaya, M. Ruben and W. Wernsdorfer, Science, 2014, 344, 1135-1138.

4 J. D. Rinehart and J. R. Long, Chem. Sci., 2011, 2, 2078-2085.

5 L. Ungur and L. F. Chibotaru, Inorg. Chem., 2016, 55, 1004310056.

6 P.-B. Jin, Y.-Q. Zhai, K.-X. Yu, R. E. P. Winpenny and Y.-Z. Zheng, Angew. Chem. Int. Ed., 2020, 59, 9350-9354.

7 J. R. Friedman, M. P. Sarachik, J. Tejada and R. Ziolo, Phys. Rev. Lett., 1996, 76, 3830-3833.

8 Y.-S. Ding, K.-X. Yu, D. Reta, F. Ortu, R. E. P. Winpenny, Y.-Z. Zheng and N. F. Chilton, Nat. Commun., 2018, 9, 3134.

9 F. Ortu, D. Reta, Y.-S. Ding, C. A. P. Goodwin, M. P. Gregson, E. J. L. McInnes, R. E. P. Winpenny, Y.-Z. Zheng, S. T. Liddle, D. P. Mills and N. F. Chilton, Dalton trans., 2019, 48, 8541-8545.

10 J. L. Liu, Y. C. Chen and M. L. Tong, Chem. Soc. Rev., 2018, 47, 2431-2453.

11 M. A. Sørensen, U. B. Hansen, M. Perfetti, K. S. Pedersen, E. Bartolomé, G. G. Simeoni, H. Mutka, S. Rols, M. Jeong, I. Zivkovic, M. Retuerto, A. Arauzo, J. Bartolomé, S. Piligkos, H.
Weihe, L. H. Doerrer, J. van Slageren, H. M. Rønnow, K. Lefmann and J. Bendix, Nat. Commun., 2018, 9, 1292.

12 M. Gregson, N. F. Chilton, A.-M. Ariciu, F. Tuna, I. F. Crowe, W. Lewis, A. J. Blake, D. Collison, E. J. L. Mclnnes, R. E. P. Winpenny and S. T. Liddle, Chem. Sci., 2016, 7, 155-165.

13 B.-C. Liu, N. Ge, Y.-Q. Zhai, T. Zhang, Y.-S. Ding and Y.-Z. Zheng, Chem. Commun., 2019, 55, 9355-9358.

14 M. Li, H. Wu, Z. Xia, L. Ungur, D. Liu, L. F. Chibotaru, H. Ke, S. Chen and S. Gao, Inorg. Chem., 2020, 59, 7158-7166.

15 M. Li, H. Wu, Z. Xia, V. Montigaud, O. Cador, B. Le Guennic, H. Ke, W. Wang, G. Xie and S. Chen, Chem. Commun., 2019, 55, 14661-14664.

16 M. J. Giansiracusa, S. Al-Badran, A. K. Kostopoulos, G. F. S. Whitehead, D. Collison, F. Tuna, R. E. P. Winpenny and N. F. Chilton, Dalton trans., 2019, 48, 10795-10798.

17 X.-L. Ding, Y.-Q. Zhai, T. Han, W.-P. Chen, Y.-S. Ding and Y.-Z. Zheng, Chem. Eur. J., doi: 10.1002/chem.202003931.

18 M. Llunell, D. Casanova, J. Cirera, J. M. Bofill, P. Alemany and S. Alvarez, SHAPE (Version 2.1), 2013.

19 D. Gatteschi, R. Sessoli and J. Villain, Molecular Nanomagnets, Oxford University Press, Oxford, 2006.

20 M. Li, H. Wu, Q. Yang, H. Ke, B. Yin, Q. Shi, W. Wang, Q. Wei, G. Xie and S. Chen, Chem. - Eur. J., 2017, 23, 17775-17787.

21 N. Ishikawa, M. Sugita and W. Wernsdorfer, Angew. Chem. Int. Ed., 2005, 44, 2931-2935.

22 P. H. Lin, T. J. Burchell, R. Clerac and M. Murugesu, Angew. Chem. Int. Ed., 2008, 47, 8848-8851.

23 T. Han, M. J. Giansiracusa, Z.-H. Li, Y.-S. Ding, N. F. Chilton, R. E. P. Winpenny and Y.-Z. Zheng, Chem. Eur. J., 2020, 26, 67736777.

24 F. Pointillart, K. Bernot, S. Golhen, B. Le Guennic, T. Guizouarn, L. Ouahab and O. Cador, Angew. Chem. Int. Ed., 2015, 54, 1504-1507.

25 E. Moreno-Pineda, M. Damjanović, O. Fuhr, W. Wernsdorfer and M. Ruben, Angew. Chem. Int. Ed., 2017, 56, 9915-9919.

26 L. F. Chibotaru, L. Ungur and A. Soncini, Angew. Chem. Int. Ed., 2008, 47, 4126-4129.

27 L. Ungur, W. Van den Heuvel and L. F. Chibotaru, New J. Chem., 2009, 33, 1224-1230.

28 G. Huang, G. Fernandez-Garcia, I. Badiane, M. Camarra, S. Freslon, O. Guillou, C. Daiguebonne, F. Totti, O. Cador, T. Guizouarn, B. Le Guennic and K. Bernot, Chem. Eur. J., 2018, 24, 6983-6991.

29 The CAMMEL code is available under GNU General Public License v3.0 and can be downloaded at https://github.com/rmarchal1/CAMME.

30 K. L. Harriman, J. L. Brosmer, L. Ungur, P. L. Diaconescu and M. Murugesu, J. Am. Chem. Soc., 2017, 139, 1420-1423.

31 T. Han, Y.-S. Ding, Z.-H. Li, K.-X. Yu, Y.-Q. Zhai, N. F. Chilton and Y.-Z. Zheng, Chem. Commun., 2019, 55, 7930-7933.

32 R. J. Blagg, L. Ungur, F. Tuna, J. Speak, P. Comar, D. Collison, W. Wernsdorfer, E. J. Mclnnes, L. F. Chibotaru and R. E. Winpenny, Nat. Chem., 2013, 5, 673-678.

33 Y. C. Chen, J. L. Liu, L. Ungur, J. Liu, Q. W. Li, L. F. Wang, Z. P. Ni, L. F. Chibotaru, X. M. Chen and M. L. Tong, J. Am. Chem. Soc., 2016, 138, 2829-2837.

34 J. Liu, Y. C. Chen, J. L. Liu, V. Vieru, L. Ungur, J. H. Jia, L. F. Chibotaru, Y. Lan, W. Wernsdorfer, S. Gao, X. M. Chen and M. L. Tong, J. Am. Chem. Soc., 2016, 138, 5441-5450.

35 S. K. Gupta, T. Rajeshkumar, G. Rajaraman and R. Murugavel, Chem. Sci., 2016, 7, 5181-5191.

36 F.-S. Guo, B. M. Day, Y.-C. Chen, M.-L. Tong, A. Mansikkamäki and R. A. Layfield, Science, 2018, 362, 1400-1403.

37 C. A. P. Goodwin, F. Ortu, D. Reta, N. F. Chilton and D. P. Mills, Nature, 2017, 548, 439-442.

38 K.-X. Yu, J. G. C. Kragskow, Y.-S. Ding, Y.-Q. Zhai, D. Reta, N. F. Chilton and Y.-Z. Zheng, Chem, 2020, 6, 1777-1793. 